\title{
Following Snowden: An International Survey
}

\author{
Kiyoshi Murata \\ Centre for Business Information Ethics, Meiji University, Tokyo, Japan \\ Andrew A. Adams \\ Centre for Business Information Ethics, Meiji University, Tokyo, Japan \\ Ana María Lara Palma \\ Civil Engineering Department, Management Area, University of Burgos, Burgos, Spain
}

\section{An International Survey on Knowledge of and Attitudes to Snowden's Revelations}

In June 2013, The Guardian in the UK and The Washington Post in the US began publishing internal electronic documents from the US' signals intelligence (SIGINT) organisation the National Security Agency (NSA), provided to them by Edward Snowden who had obtained the documents while employed as a systems administrator at the NSA for contractor Booz Allen Hamilton. As they have done previously, the NSA and other parts of the US government generally will not confirm or deny the validity of the documents, however on 21 st June 2013, the US Department of Justice charged Snowden with violating the Espionage Act. The activities detailed in the documents included activity undertaken by the NSA and its main SIGINT partner the UK's Government Communications Headquarters (GCHQ), and with the SIGINT agencies of three former British colonies (Canada, Australia and New Zealand), as well as joint activities with similar agencies in other countries such as Germany's Bundesnachrichtendienst (BND).

In 2014, the Pew Research Center (Madden, 2014) undertook the first of a number of surveys of US citizens' attitudes to Snowden and the documents he revealed. In particular, they asked questions such as whether respondents believed that Snowden's revelations had served or harmed the public good, whether Snowden should be prosecuted or not. Inspired by these surveys, a group of academics at Meiji University in Tokyo developed a pilot survey deployed in Japan and Spain using students as the primary research population (for reasons of resource constraints) and conducted follow-up interviews. The results of this pilot survey are presented in Murata, Adams and Lara Palma (2017). Having revised the survey after analysis it was deployed with the cooperation of local academics in Mexico, New Zealand, Spain and Sweden (in English), and in translation in Japan and Germany. With the aid of graduate students studying in Tokyo, it was also translated into Chinese and deployed in Taiwan (using traditional Chinese characters) and the People's Republic of China (using simplified Chinese characters). The choice of countries was a combination of deliberation and pragmatism. The following countries had suitable resources available: New Zealand was chosen as a Five Eyes member; Germany, Spain and Sweden provide an EU perspective; Mexico provides a US neighbouring perspective as well as a Spanish-influenced culture outside Spain; and Japan, China 
and Taiwan provide a South East Asian viewpoint. This paper presents the original English language version of the survey which was deployed, or which was translated into a local language for deployment.

\section{A survey on privacy awareness}

This questionnaire survey is conducted by [names of local project members who conducted the survey with their affiliations] as part of a cross-national study on the privacy awareness of young people. The survey outcomes will be statistically analysed and interpreted based on the purpose of the study only (to clarify similarities and differences in university students' privacy awareness between nations/regions) and will never be used for other purposes. Neither the survey outcomes nor the results of the analysis will be made public in any way that allows an individual respondent to be identified. The questionnaire contains questions about technical issues and current affairs/news items. Please try to answer as honestly and frankly as possible, within the limits of your knowledge. It will take 20-30 minutes to complete responding to the questionnaire. Many thanks for your time and cooperation.

If you have any question about the survey, please don't hesitate to contact with [contact details of a local project team].

\section{Q1. Gender}

1. Male; 2. Female

\section{Q2. Age}

1. $18 ; 2.19 ; 3.20 ; 4.21 ; 5.22 ; 6.23 ; 7.24 ; 8.25+$

\section{Q3. Affiliation}

[A list of universities]

\section{Q4. Primary area of study}

1. humanities; 2. law; 3. political science; 4. economics; 5. commerce/business administration; 6. sociology; 7. natural/physical science; 8. technology/engineering; 9. medicine/nursery/health science; 10. agriculture; 11. education; 12. policy making; 13. informatics; 14. not yet determined; 15. other...Please specify [ ]

\section{Q5. Nationality}

[A list of countries] 


\section{Q6. Which ethnic group do you belong to? (optional)}

[A list of ethnic groups]

\section{Q7. Do you feel that your use of the Internet involves taking risks with your privacy?}

1. Strongly; 2. To an extent; 3. Not much; 4. Not at all; 5. Prefer not to answer

\section{Q8. Do you feel that your non-Internet activity involves taking risks with your privacy?}

1. Strongly; 2. To an extent; 3. Not much; 4. Not at all; 5. Prefer not to answer

\section{Q9. How much do you feel that the following groups threaten your privacy?}
a. Individuals who you know well
b. Individuals who you know but not well
c. Individuals who you don't know
d. Telecom companies/ Internet providers ([names of telecom companies/Internet providers])
e. Internet companies (Google, Twitter, Facebook, Yahoo!, [names of local Internet companies], etc.)
f. Computer hardware companies (Intel, Lenovo, HP, Dell, etc.)
g. Computer software companies (Microsoft, Oracle, etc.)
h. System Integrators
i. Other for-profit companies
j. Health-care organisations
k. Educational institutions
1. Other not-for-profit organisations
$\mathrm{m}$. Law enforcement government agencies (Police, [names of local agencies], etc.)
n. Secret service government agencies ([names of local agencies], etc.)
o. Other government agencies ([names of local agencies in charge of health, interior and tax], etc.)

1. Very much; 2. To an extent; 3. Not much; 4. Not at all; 5. Have not heard of this group; 6. No opinion; 7. Prefer not to answer

\section{Q10. How much do you feel that the following technologies threaten your privacy?}
a. CCTV
b. GPS (Global Positioning System)
c. ETC (Electronic Toll Collection)
d. Smart meter (an electricity meter providing your supplier with regular, approx. every 30 minutes, readings of your usage)
e. Home automation which senses human activities (e.g., air conditioner, lighting apparatus) 
f. Personal computer (Widows machines, Mac, etc.)

g. Smart phone (iPhone, Android, etc.)

h. Home video game console (Wii, PlayStation, XBOX, etc.)

i. Portable video game console (PSP, Wii-U, etc.)

j. Smart card ([names of local smart cards], etc.)

k. RFID (Radio Frequency Identification)

1. Home-based health monitoring (e.g., a smart toilet which automatically performs urinalysis)

m. Personal body monitoring (Fitbit, etc.)

n. Behavioural targeting

o. Social media services

p. Online shopping (B to $\mathrm{C}$ ecommerce)

q. Online auction

r. Online games

s. Making payments online

1. Very much; 2 . To an extent; 3 . Not much; 4 . Not at all; 5 . Have not heard of this technology; 6. No opinion; 7. Prefer not to answer

If there are any other technologies that pose a significant or greater threat to your privacy, please specify [ ]

\section{Q11. Is your right to privacy important?}

1. Very important; 2. Important; 3. Not so important; 4 . Not important at all; 5 . Prefer not to answer

\section{If 1 or 2 to Q11:}

Q12. Please describe why your right to privacy is important. [ ]

\section{If 3 or 4 to Q11:}

Q13. Please describe why your right to privacy is not important. [ ]

\section{Q14 How well do you understand what the right to privacy is?}

1 Understand very well; 2 Understand; 3 Hardly understand; 4 Don't understand at all; 5 Prefer not to answer

\section{If 1 or 2 to Q14:}

Q15. Please describe what the right to privacy is. [ ] 
Q16. Do you know much about the following organisations?
a. FBI (Federal Bureau of Investigation)
b. CIA (Central Intelligence Agency)
c. NSA (National Security Agency)
d. GCHQ (Government Communications Headquarters)
e. [names of local secret service government agencies]
f. [names of local privacy commissioner offices]

1. I've heard of it and understand it; 2. I've heard of it but don't understand it; 3. I haven't heard of it; 4. Prefer not to answer

Q17. Have you heard about US Army Private First Class Bradley (Chelsea) Manning revealing large amounts of US classified data through Julian Assange's Wikileaks in 2010?

[picture of Manning as of 2010 leaks]; [picture of Assange]

1. Yes; 2. No; 3. Prefer not to answer

\section{If 1 to Q17:}

Q18. How much do you know about those revelations?

1. A lot; 2. A fair amount; 3. Not much; 4. I've only heard about it; 5. Prefer not to answer

Q19. Did Manning's revelations serve the public interest or harm it?

1. Served it a lot; 2. Served it to an extent; 3. Harmed it to an extent; 4. Harmed it a lot; 5. No opinion; 6. Prefer not to answer

Q20. Have you heard about former NSA contractor Edward Snowden revealing large amounts of information about the activities of the NSA and GCHQ, through the UK's The Guardian newspaper and the US's The Washington Post newspaper starting in June 2013?

[picture of Snowden]

1 Yes; 2 No; 3 Prefer not to answer

\section{If 1 to Q20:}

Q21. How did you get and have updated your knowledge about those revelations? (Multiple answers allowed)

1. Read newspaper articles; 2. Watched TV news reports; 3. Read news reports on the Internet; 4. Through social media; 5 . Heard during lectures at my university; 6 . Heard from your friends or acquaintances; 7. Prefer not to answer; 8. Others [ ] 
Q22. Have you ever talked about those revelations with others?

1. Yes; 2. No; 3. Prefer not to answer

\section{Q23. Have you ever searched for information about those revelations?}

1. Yes; 2. No; 3. Prefer not to answer

Q24. How much do you know about the contents of those revelations?

1. A lot; 2. A fair amount; 3. Not much; 4. Little; 5. Prefer not to answer

Q25. Have you changed your way of communicating online using systems such as social media (e.g., Twitter, Facebook), YouTube, blogging, Skype, email and instant messaging since you heard about Snowden's revelations? (Multiple answers allowed)

1. Have not changed at all; 2. Stopped using some systems; 3 . Have tried to cut down my use of some systems; 4. Have deleted (some of) personal data and contents I had posted on social media; 5. Have paid more attention to personal data and contents posted on social media; 6 . Have changed my privacy settings on some systems; 7. Prefer not to answer; 8. Other changes [ ]

Q26. How much do you know about the US government's reactions to those revelations?

1. A lot; 2. A fair amount; 3. Not much; 4. Little; 5 . Prefer not to answer

Q27. How much do you know about the current status of Mr Snowden?

1. A lot; 2. A fair amount; 3. Not much; 4. Little; 5. Prefer not to answer

Please read the story of "Edward Snowden's Revelations" then respond to the following questions.

"Edward Snowden's Revelations"

On 5th June 2013, a bylined article titled "NSA collecting phone records of millions of Verizon customers daily" was published on the website of British newspaper The Guardian [1]. The article stated that the telephone records of millions of US customers of Verizon, one of America's largest telecoms providers, were being collected on an "ongoing, daily basis" by the NSA (National Security Agency), an intelligence agency of the US Department of Defence, regardless of whether they were suspected of any wrongdoing, under a top secret court order issued in April 2013. The next day, a picture of the PRISM program, which had been operated by the NSA since 2007, was reported on 
the websites of US newspaper The Washington Post and The Guardian with the Power Point slides describing the program which were classified documents of the NSA [2]. According to the reports, this program allowed the NSA to monitor individual users, not only in the USA but also throughout the world, indiscriminately and in bulk by collecting their communication data such as email contents, search history, live chat and transferred files. It did this through directly accessing the servers of US IT companies including Microsoft, Google, Yahoo!, Facebook, Apple, YouTube and Skype. The Boundless Informant program which was operated by the NSA to collect, analyse and store billions of data of emails and phone calls passing through the US communication infrastructures was also reported online [3]. The subsequent media reports revealed that the NSA and its British counterpart the GCHQ (Government Communications Headquarters) had carried out effectively limitless information gathering and indiscriminate mass monitoring.

Four days after the first report, an interview video with the person who provided these details to the newspapers was released on the Guardian's website [4]. His name was Edward Snowden, a 29-yearold employee of the NSA's contractor Booz Allen Hamilton. Throughout his work at US intelligence agency the CIA (Central Intelligence Agency), Dell, another NSA contractor, and Booz Allen Hamilton, he collected the classified information that was the material of the media reports to reveal the whole picture of monitoring conducted by the NSA all over the world.

On 21st June the US government filed charges of spying against Snowden who at that point was in Hong Kong, and then revoked his passport the next day. On 23rd July, however, he flew to Moscow where he is currently living in exile. His revelations have attracted heavy doses of both praise and censure; whereas some have positively evaluated his deed as an act of valour to protect democracy against the tyranny of the state, others have criticised him as a traitor to his country that have been preoccupied with responses to the threat of terrorism since the 9.11 attacks. His revelations have generated lively discussions of national security, safety and security of societies, personal freedom and privacy in the world.

\section{References}

[1] http://www.theguardian.com/world/2013/jun/06/nsa-phone-records-verizon-court-order

[2] http://www.washingtonpost.com/wp-srv/special/politics/prism-collection-documents/ http://www.theguardian.com/world/2013/jun/06/us-tech-giants-nsa-data

[3] http://www.theguardian.com/world/2013/jun/08/nsa-boundless-informant-global-datamining?

$\mathrm{CMP}=$ twt $\mathrm{gu}$

[4] http://www.theguardian.com/world/video/2013/jun/09/nsa-whistleblower-edward-snowdeninterview-video 
Q28. Why do you think Snowden determined to make those revelations? [ ]

Q29. Have Snowden's revelations served the public interest or harmed it?

1. Served it a lot; 2. Served it to an extent; 3. Harmed it to an extent; 4. Harmed it a lot; 5. No opinion; 6. Prefer not to answer

Q30. Should the US government pursue a criminal case against Snowden?

1. Should do; 2. Should not do; 3. No opinion; 4. Prefer not to answer

Q31. If you were an American citizen and were faced with a similar situation to Snowden, do you think you would do what he did?

1. Yes; 2. No; 3. Prefer not to answer

If 1 to Q31:

Q32. Why would you do what Snowden did if you were an American citizen and were faced with a similar situation to him? [ ]

If 2 to Q31:

Q33. Why would you not do what Snowden did if you were an American citizen and were faced with a similar situation to him? [ ]

Q34. If you were faced with a similar situation to Snowden in your country, i.e. you found out that a [local country's] intelligence agency was conducting similar operations to those of the NSA and GCHQ, would you, as a [local country's] citizen, do what he did?

1. Yes; 2. No; 3. Prefer not to answer

If 1 to Q34:

Q35. Why would you do as Snowden did if you were faced with a similar situation to Snowden in your home country? [ ]

If 2 to Q34:

Q36. Why would you not do as Snowden did if you were faced with a similar situation to Snowden in your home country? [ ]

Q37. What social changes do you think have happened because of Snowden's revelations?

1. No social change has happened; 2. No opinion; 3. Prefer not to answer; 4. The following social 
changes have happened [ ]

Q38. How much do you feel that individuals in your country must give up privacy and freedom in order to ensure safety and security of the society and individuals?

1. Very Much; 2. To an extent; 3. Not much; 4. Not at all; 5. No opinion; 6. Prefer not to answer

\section{Thank you for your cooperation!}

If you are willing to be interviewed in more depth about these issues, please provide your name and a contact email address - we will approach a few of our willing respondents for interview. We'd like to ask you to provide your name and email address if you accept our follow-up interviews.

Name [ ]; Email [ ] 


\section{Acknowledgements}

This study was supported by the MEXT (Ministry of Education, Culture, Sports, Science and Technology, Japan) Programme for Strategic Research Bases at Private Universities (2012-16) project "Organisational Information Ethics" S1291006 and the JSPS Grant-in-Aids for Scientific Research (B) 24330127 and (B) 25285124.

\section{References}

Madden, M. (2014). "Public Perceptions of Privacy and Security in the Post-Snowden Era", available at http://www.pewinternet.org/2014/11/12/public-privacy-perceptions/ (accessed 21st March, 2017).

Murata, K., Adams, A. A., and Lara Palma, A. M. (2017) "Following Snowden: A Cross-cultural Study on the Social Impact of Snowden's Revelations", Journal of Information, Communication and Ethics in Society, Vol. 15 No. 3, pp ??-?? 